\title{
Ureteral injury during malignant gynecologic surgery: A single center retrospective study
}

\author{
Ying Han ${ }^{1}$, Yan Zhai ${ }^{1, *}$ and Shuzhen Wang ${ }^{1}$ \\ ${ }^{1}$ Department of Obstetrics and Gynecology, Beijing Chao-Yang Hospital, Capital Medical University, Beijing, P. R. China \\ *Correspondence: zhaiyan767@sina.com (Yan Zhai)
}

DOI: $10.31083 /$ i.jmcm.2020.03.002

This is an open access article under the CC BY 4.0 license (https://creativecommons.org/licenses/by/4.0/).

Ureteral injury is common in gynecologicy oncology operations. important to understand its characteristics. From January 2000 to December 2017, a total of 2041 patients were diagnosed with malignant gynecology diseases and underwent surgery in the Department of Obstetrics and Gynecology, Beijing Chao-Yang Hospital, Capital Medical University. All the medical records were retrospectively reviewed and 27 cases of ureteral injury were identified. The type of gynecologic oncology surgery, the site and the type of ureteral repair and the related morbidity were analyzed. Total number of ureteral injury was 27 (1.32\%) out of 2041 patients underwent gynecologic oncology operations. Of the 2041 patients, 1029 had cervical cancer, 401 harbored ovarian cancer and 611 were diagnosed with endometrial cancer. Of all the procedures analyzed, we found 19 cases of radical cervical cancer surgery $(1.84 \%), 5$ cases of ovarian cancer surgery $(1.25 \%)$ and 3 cases of endometrial cancer surgery $(0.49 \%)$. Ureteral injuries were recognized intraoperatively in 9 patients $(33.3 \%)$ and postoperatively in 18 patients $(66.7 \%)$ with a mean delay of 7.8 days (range from 3 to 21 days). The type of ureteral injuries included laceration $3 / 27$ (1 1.1\%), partial or total accidental transection $2 / 27(7.4 \%)$, partial resection due to tumor infiltration $7 / 27(25.9 \%)$ and fistula formation 15/27 (55.6\%). Ureteral repair surgeries contained $7 / 27$ (25.9\%) ureterostomy, 15/27 (55.6\%) insertion of Double-J stent and 5/27 (18.5\%) ureteral reconnection. Febrile $(11 / 27,40.7 \%)$ and leukocytosis $(11 / 27$, $40.7 \%$ ) were the most common postoperative symptoms. Ureteral injury is a common complication in gynecologic oncology operations. Immediate recognition and treatment based on appropriate guide lines result in good outcome.

\section{Keywords}

Gynecologic oncological surgery; ureteral injury; morbidity

\section{Introduction}

In gynecologic oncology operations, lesions of the urinary tract are common complications. Of urinary injuries, ureteral injury has the highest incidence. Despite the progress in surgical techniques, especially laparoscopic surgery, ureteral injury continues to be a serious complication in patients who undergo gynecologic oncology surgery, and most of them require surgical repair.
After reviewing the literature, we found that about $57-82 \%$ of ureteral injuries are associated with gynecologic oncology procedures, especially radical hysterectomy [1]. The fraction of ureteral injury which took place in surgical procedures of gynecologic oncology diseases was about 0.4 to $2.5 \%$ [2]. Only one-third of these injuries were detected immediately during surgery [2]. Ureteral injuries seriously affect the postoperative quality of patients regarding complications such as severe pain, fever, infection and more. Therefore, it is necessary to detect those lesions immediately and treat them as soon as possible. The objective of our study was to investigate the characteristics and treatment of ureteral injuries in gynecologic oncology surgery in the department of gynecology oncology, Beijing Chaoyang hospital, over a period of 17 years.

\section{Materials and Methods}

We reviewed the medical records of the patients in this period. The including criteria were: 1) Patients with gynaecologic oncology surgery who were identified with ureteral lesions during or after surgery between January 2000 to December 2017; 2) Patients who received a surgical intervention for ureteral repair during the primary surgery or in a second procedure.

Our current report represents a retrospective study. All study procedures were approved by the ethics committee of the Beijing Chao-Yang Hospital, Capital Medical University (No. 2017354). We conducted research using identifiable electronic medical record data. The research project did not involve personal privacy and commercial interests and was no longer accessible to participants. Therefore, the ethics committee approved the waiver of signed written informed consent and approved the procedure for verbal consent. We retrospectively analyzed the medical data records. All patients in this study gave their verbal informed consent at the time of diagnosis that their medical records could be reviewed within the retrospective studies.

Of all the medical records, gynecologic oncology surgical procedures were performed by gynecologic oncologists and the surgical repair of ureter in cooperation with a urologist. We analyzed all the data to determine the type, the location and the frequency of ureteral injuries, the type of operative procedure,the time of diagnosis,the length of hospitalization and the postoperative complications such as fever over $38{ }^{\circ} \mathrm{C}$, urinary tract infection,wound infection and secondary ureteral stenosis. We used the software SPSS 22.0 (Chicago, IL) to analyze the statistical significance of the data. 
Table 1. Clinicopathology characteristics of the 27 Patients.

\begin{tabular}{lc}
\hline Parameter & \\
\hline Age in years (mean \pm SD) (range) & $49.33(27-74)$ \\
\hline Weight in kg (mean \pm SD) (range) & $60.10(44-72)$ \\
\hline Initial diagnosis & Number of patients [n/N (\%)] \\
Cervical cancer & $19 / 1029(1.84 \%)$ \\
Endometrial cancer & $3 / 611(0.49 \%)$ \\
Ovarian cancer & $5 / 401(1.25 \%)$ \\
Gynecologic procedure & \\
Laparotomy & \\
Simple TAH & \\
Radical TAH & $1 / 27(3.7 \%)$ \\
Primary debulking & $8 / 27(29.6 \%)$ \\
Secondary debulking & $2 / 27(7.4 \%)$ \\
Laparoscopy & $3 / 27(11.1 \%)$ \\
Simple TAH & \\
Radical TAH & \\
Type of ureteral injury & $2 / 27(7.4 \%)$ \\
Laceration & $11 / 27(40.7 \%)$ \\
Metastasectomy & \\
Transection total or partial & $3 / 27(11.1 \%)$ \\
Fistula & $7 / 27(25.9 \%)$ \\
Location of ureter injury & $2 / 27(7.4 \%)$ \\
Distal & $15 / 27(55.6 \%)$ \\
Proximal & \\
& \\
\hline & \\
\hline & \\
\hline
\end{tabular}

\section{Results}

From January 2000 to December 2017, a total of 2041 cases of gynecologic oncology operations were performed in our center. Of all these patients, $27(1.32 \%)$ received an intervention for ureteral repair during or after the initial surgery. The patients characteristics, the type of the operations and information of ureteral repair are presented in Table 1. There were $19(1.84 \%)$ of ureteral injury cases out of 1029 cervical cancer radical surgery, 5 (1.25\%) ureteral injuries out of 401 ovarian cancer surgery as well as 3 $(0.49 \%)$ out of 611 endometrial cancer surgery cases.

The different types of ureteral injuries $(n=27)$ were: (1) Metastatic lesions (7, 25.9\%) involving ureter resection during ovarian cancer debulking surgery; (2) Laceration (3, 11.1\%) during surgery like radical dissection or lymphadenectomy; (3) Partial and total transection $(2,7.4 \%)$ during pelvic tumor excision either at the level of infundibulopelvic ligament or uterine artery; (4) Urinary fistulas $(15,55.6 \%)$ due to secondary ischemic necrosis after accidental ligation or coagulation of ureteral vessel during radical hysterectomy or as a late presentation of the ureter's unintended ligation during simple hysterectomy. Of all the cases, the distal part of the ureter was involved in $18(66.7 \%)$ cases at the level of crossing the uterine artery or at the insertion into bladder. Moreover, 9 cases $(33.3 \%)$ were proximal part at the level of the infundibulopelvic ligament.

In our study, the ureteral injury was unilateral in all cases. Ureteral injuries were recognized during the procedure in 9 (33.3\%) patients and afterwards in $18(66.7 \%)$ patients with a mean delay of 7.3 days (range from 3 to 21 days), depending on the presenting clinical picture. The repair surgical procedures and the postoperative symptoms or complications are presented in Ta- ble 2. Of all the patients, ureteroneocystostomy was performed in 7 patients (25.9\%), insertion of Double-J stent was conducted in $15(55.6 \%)$ patients and ureteroureterostomy was carried out in 5 (18.5\%) patients. The most common postoperative symptoms of the patients was febrile morbidity $(11 / 27,40.7 \%)$ and leukocytosis $(11 / 27,40.7 \%)$, followed by ileus $(2 / 27,7.4 \%)$ and wound infection $(1 / 27,3.8 \%)$. Furthermore, hydronephrosis was found by ultrasound in $2(7.4 \%)$ patients. There was no significant difference in age, body weight, hospital stay, surgeon experience and morbidity in terms of fever, leukocytosis and wound infection were seen between early and late operative procedures for ureteral repair. All the cases were followed up with ultrasound and blood test and the final outcome was successful.

Table 2. Surgical management, postoperative symptoms or complications of ureteral injuries.

\begin{tabular}{lc}
\hline Type of surgery [N(\%)] & \\
\hline Ureteroureterostomy & $5(18.5 \%)$ \\
\hline Double-j insertion & $15(55.6 \%)$ \\
Ureteroneocystostomy & $7(25.9 \%)$ \\
\hline Hospitalization in days (mean \pm SD) (range) & $12.2(6-19)$ \\
Postoperative symptoms or complications [N(\%)] & \\
Wound infection & $1(3.8 \%)$ \\
Fever & $11(40.7 \%)$ \\
Leukocytosis & $11(40.7 \%)$ \\
Hydronephrosis & $2(7.4 \%)$ \\
Ileus & $2(7.4 \%)$ \\
Follow-up in months (mean \pm SD) (range) & $67(2-187)$ \\
\hline
\end{tabular}

\section{Discussion}

It is well known that ureteral injury is relatively common in gynecologic oncological surgeries, especially in cervical carcinoma and ovarian cancer. The underlying basis for this is that the female reproductive system is adjoined to the urinary organs. Especially, ureteral damage at the level of in fundibulopelvic ligament or uterine artery [3]. It was reported that about $50 \sim 80 \%$ ureteral injuries in gynecologic oncological surgeries are due to the anatomic proximity of the structures. In the recent years, with the development of micro-invasive techniques, the use of energy instruments and more general and stringent radical surgery were related with the increasing ureteral injuries [4].

By reviewing the related articles, we know that the rate of ureteral injury was between 0.05 and $0.5 \%$, while the rate of bladder injury in hysterectomy surgery was 0.5 to $2 \%$ [5]. And interestingly, another article stated that the rate of surgery related ureteral and bladder injuries must be higher than reported for many cases were diagnosed later [6]. The results of our study showed that the ureteral injury incidence rate of gynecological oncology surgery is the same with reported data.

In our present study, there was no difference in prior abdominal surgery, body mass index or postoperative pathologic findings. It has been shown previously that the most common location of ureteral repair was in the distal $3 \mathrm{~cm}$ of the ureter. Moreover, the type of ureteral injuries included laceration, metastasectomy, 
transection total or partial fistula. We could see that the injuries occurring nearly to the pelvis, which happened during the ovarian tumor dissection, oophorectomy and lymphadenectomy, while occurring distally at the level of uterosacral ligament during dissection in the pouch of Douglas and at the cardinal ligament during radical hysterectomy [7, 8]. It is reported that approximately $30 \%$ of patients received ureteral repair at the time of primary surgery [1]. In our study, ureteral injuries were recognized intraoperatively in 9 patients (33.3\%) and postoperatively in 18 patients $(66.7 \%)$. In the time after surgery, it is necessary to evaluate immediately when suspicious complications presented [9]. When unilateral ureteral obstruction happened in the first 24 to 48 hours after surgery, serum creatinine level may show a transient increase [8]. All the cases in our study were treated within 1 month to minimize the worse complication such as kidney loss.

In our center, all 27 patients received active surgical repair treatment by a senior urologist. Ureteroneocystostomy was performed in about $25.9 \%$ patients,insertion of Double-J stent was conducted in about $55.6 \%$ patients and ureteroureterostomy was undertaken in $18.5 \%$ patients. If we could diagnose an injury within the first 24 to 48 hours, an repair surgery could be performed right now [10, 12]. In fact, most cases were diagnosed later, and the chance to perform immediate surgery reduced because of the necrosis, edema, and tissue reaction. All the cases in our study were treated within 1 month to minimize the worse complication. In all cases,patients were diagnosed with ureteral injury by CT pye-lography with failure of double-J stent placement by cystoscopic retrograde. There was viewpoint that supporting tunneling the ureter at implantation $[7,11]$ in the bladder to create an anti-reflux mechanism, however, all of our cases were managed with reflux implantation to avoid possible stenosis at the implantation site.

Ureteral injury is a common complication in gynecologic oncology operations. Immediate recognition and treatment based on appropriate pricinple is very important.

\section{Availability of data and materials}

The datasets used or analyzed during the current study are available from the corresponding author upon request.

\section{Ethics Approval and Consent to Participate}

Our report represents a retrospective study. All study procedures were approved by the ethics committee of the Beijing ChaoYang Hospital, Capital Medical University (No.2017-354). The clinical registration number is ZYLX201713. We conducted research using identifiable electronic medical record data. The research project did not involve personal privacy and commercial interests and was no longer accessible to participants. Therefore, the ethics committee approved the waiver of signed written informed consent and approved the procedure for verbal consent. We retrospectively analyzed the medical data records. All patients in this study gave their verbal informed consent at the time of diagnosis that their medical records could be reviewed within the retrospective studies.

\section{Authors' contributions}

YZ and YH designed the study and collected the patients' data. SZ Wanalyzed the data and performed statistical analysis. YH was a major contributor in writing the manuscript. All authors read and approved the final manuscript.

\section{Acknowledgments}

We would like to express my gratitude to all those who helped me during the riting of this manuscript.

Thanks to all the peer reviewers and editors for their opinions and suggestions.

\section{Comflict of Interest}

The authors declare that they have no competing interests.

Submitted: April 22, 2020

Accepted: September 16, 2020

Published: September 20, 2020

\section{References}

[1] Rock J, Jones A, Howard W. Operative injuries to the ureter: prevention, recognition and management. In: The Linde's operative gynecology, 10th edition. Williams \& Wilkins, Philadel-phia. 2008; 961971.

[2] ACOG Technical Bulletin. Lower urinary tract operative injuries. International Journal of Gynecology \& Obstetrics. 1997; 5(1): 67-72.

[3] Gilmour DT, Baskett TF. Disability and litigation from urinary tract injuries at benign gynecologic surgery in Canada. Obstet Gynecol. 2005; 105(1): 109-114.

[4] Assimos DG, Patterson LC, Taylor CL. Changing incidence and etiology of iatrogenic ureteral injuries. J Urol. 1994; 152(6 Pt 2):22402246.

[5] Hill DJ. Complications of hysterectomy. Bailliere's Clin Obstet Gynaecol. 1997; 11(1): s181-197.

[6] Gilmour DT, Dywer PL, Carey MP. Lower urinary tract injury during gynecologic surgery and its detection by intraoperative cystoscopy. Obstet Gynaecol. 1999; 94(5 Pt 2): 883-889.

[7] Carley ME, McIntire D, Carley JM, Schaffer J. Incidence, risk factors and morbidity of unintended bladder or ureter injury during hysterectomy. Int Urogynecol J. 2002; 13(1): 18-21.

[8] Aronson MP, Bose TM. Urinary tract injury in pelvic surgery. Clin Obstet Gynecol. 2002; 45(2): 428-432.

[9] Savan K, Ekin M, Kupelioglou L, Oral S, Yasar L. Surgical repair of genitourinary fistulae: comparison of our experience at Turkey and Niger. Arch Gynecol Obstet. 2010; 282(6): 649-653.

[10] Rosen DMB, Korda AR, Waugh RC. Ureteric injury at Burch colposuspension: 4 case reports and literature review. Aust NZ J Obstet Gynaecol. 1996; 36(3): 354-358.

[11] Deutsche Gesellschaft für Gynäkologie und Geburtshilfe (DGGG), Arbeitsgemeinschaft Medizinrecht (2010) Operationsbedingte Verletzungen des Ureters in der Gynäkologie und Geburtshilfe.

[12] Rao D, Yu H, Zhu H, Duan P. The diagnosis and treatment of iatrogenic ureteral and bladder injury caused by traditional gynaecology and obstetrics procedures. Arch Gynecol Obstet. 2012; 285(3): 763765 . 